\title{
EchoGéo
}

42 | 2017

Varia

\section{La fiction spéculative sud-africaine : une littérature de genre au service d'une critique de la société sud- africaine contemporaine}

\section{Sophie Didier}

\section{CpenEdition}

Journals

Édition électronique

URL : https://journals.openedition.org/echogeo/15157

DOI : 10.4000/echogeo.15157

ISSN : 1963-1197

Éditeur

Pôle de recherche pour l'organisation et la diffusion de l'information géographique (CNRS UMR 8586)

Référence électronique

Sophie Didier, «La fiction spéculative sud-africaine : une littérature de genre au service d'une critique de la société sud-africaine contemporaine », EchoGéo [En ligne], 42 | 2017, mis en ligne le 31 décembre 2017, consulté le 31 juillet 2021. URL : http://journals.openedition.org/echogeo/15157 ; DOI : https:// doi.org/10.4000/echogeo.15157

Ce document a été généré automatiquement le 31 juillet 2021.

EchoGéo est mis à disposition selon les termes de la licence Creative Commons Attribution - Pas d'Utilisation Commerciale - Pas de Modification 4.0 International (CC BY-NC-ND) 


\title{
La fiction spéculative sud-africaine : une littérature de genre au service d'une critique de la société sud- africaine contemporaine
}

\author{
Sophie Didier
}

1 Le texte publié ci-dessous repose sur la lecture des romans suivants :

2 - Arigbabu (ed.), Lagos_2060 : exciting sci-fi stories from Nigeria, Lagos: DADA Books/Design and Dream Arts Enterprises, 2013.

- L. Beukes, Zoo City, Johannesburg: Jacana, 2010. Traduit de l'Anglais en français en 2011 par Laurent Philibert-Caillat, Paris: Eclipse. Édition Poche 2016 aux Presses de la Cité.

- S.L. Grey, The Mall (downside), Londres: Corvus/Atlantic Books, 2011, édition Kindle.

- I. Hartmann (ed.), AfroSF: Science Fiction by African Writers, Storytime, 2012, édition Kindle.

- H. Rose-Innes, Nineveh, Le Cap: Umuzi, 2011, édition Kindle. Traduit de l'Anglais en Français par Elisabeth Gilles, Carouge : Zoé, 2014.

3 Un intéressant mouvement littéraire anime depuis une petite dizaine d'années plusieurs pays africains anglophones sur le front pourtant très "occidental" de la science-fiction. L'Afrique du Sud, en partie pour des raisons assez logiques liées au marché du livre dans ce pays, est aux avant-postes de ce renouvellement de genre de la littérature africaine, et cet article vise à présenter quelques exemples sud-africains représentatifs pour la façon dont ils traitent le moment post-apartheid à travers ce genre très particulier ${ }^{1}$.

La montée en puissance de la science-fiction en Afrique du Sud, mais aussi au Nigéria, a ceci de remarquable qu'elle s'appuie sur les nouveaux moyens de diffusion à la disposition des éditeurs et des auteurs, moyens qui permettent de réduire les coûts de production et surtout d'augmenter la disponibilité des titres : les blogs bien sûr, mais aussi les micro-presses et les e-books, qui permettent une diffusion et une mise en 
réseau des auteurs au-delà de leurs frontières nationales, et qui représentent peut-être aussi une forme de prise d'indépendance vis-à-vis des grands bailleurs internationaux qui soutiennent financièrement les éditeurs du continent. C'est en tous les cas le modèle proposé par l'anthologie de nouvelles de science-fiction africaine Afro-SF réunie par Ivor Hartmann, auteur et éditeur zimbabwéen basé en Afrique du Sud (Hartmann, 2012). Pour Hartmann, écrire de la science-fiction en Afrique n'a rien d'une inféodation à des genres et des modes d'écriture américains ou britanniques, qui garantirait aux auteurs africains le succès mais les couperaient de leur base (de fait, le succès commercial n'est pas encore vraiment au rendez-vous pour tous ces auteurs). Il s'agit bien pour lui de se réapproprier le futur du continent grâce à la science-fiction, pour pouvoir en maitriser le développement futur :

"SciFi is the only genre that enables African writers to envision a future from our African perspective. (...) If you can't see and relay an understandable vision of the future, your future will be co-opted by someone else's vision, one that will not necessarily have your best interests at heart" (Hartmann, 2012, loc. 93).

On rejoint ici les commentaires de Middleton et Woods sur le rôle qu'endosse la science-fiction par rapport au temps présent, ce genre ne relevant évidemment pas d'une invention pure, mais plutôt d'un méta-commentaire sur la réalité actuelle (Middleton \& Woods, 2000). Le tour post-colonial est évidemment en plus ici, soulignant la nécessaire réappropriation du genre contre la confiscation des voix africaines.

Le point de vue de Hartmann n'est pas isolé : dans l'anthologie nigériane Lagos_2060 pilotée par Ayodele Arigbabu (lui-même auteur précédemment publié dans le recueil Afro-SF sus-nommé), les nouvelles, publiées pour l'essentiel par des amateurs, renvoient à des anticipations possibles de la métropole du Nigéria, et parlent évidemment beaucoup de corruption et de faillite politique, ce qui n'est pas à proprement parler un scoop pour le Nigéria. Mais elles parlent aussi de perte de repères culturels dans la mondialisation et de crise sociale et écologique majeure : l'une de ces nouvelles met en scène une société nigériane fictive de biotechnologie responsable de la création de terrifiantes grenouilles mutantes, une autre recrée un méga-projet urbain proche de l'actuel projet Eko Atlantic ${ }^{2}$ au service d'une dénonciation des nouvelles formes de ségrégation socio-spatiale qui recomposent Lagos au gré des investissements massifs dans l'immobilier accompagnant la croissance économique de la grande métropole nigériane... Dans l'urgence des transformations actuelles de la ville et de l'accélération de son ancrage à la mondialisation, la science-fiction apparaît dès lors comme un moyen très efficace de capter et de mettre en forme les inquiétudes populaires vis-à-vis des dynamiques urbaines contemporaines du Nigéria.

\section{Fiction spéculative en Afrique du Sud : essai de définition et archétype du genre}

7 Pour ce qui concerne l'Afrique du Sud, le genre de la fiction spéculative est peut-être moins directement associé au renouveau de la littérature que le polar. Ces ouvrages sont en effet rapidement traduits et sont généralement des succès de librairie, à l'exemple des productions de Deon Meyer, écrits en anglais et en afrikaans et publiés aux éditions du Seuil depuis le début des années 2000. Outre Meyer, d'autres auteurs font aujourd'hui partie de ce paysage du polar sud-africain, comme Louis-Ferdinand 
Despreez publié chez Phébus, ou Margie Orford chez Payot... Ces romans, en mettant face à face des policiers fatigués et des tueurs en série ou des gangs ultra-violents du Cap et de Johannesburg, permettent à leurs auteurs de prendre la mesure du chemin parcouru (ou pas) depuis la fin de l'apartheid, en termes notamment de rapports de race, pour des auteurs qui restent dans leur grande majorité d'origine blanche anglophone ou afrikaaner ${ }^{3}$. À travers le genre du polar, ce sont bien les enjeux identitaires de la nouvelle Afrique du Sud qui sont ici questionnés, et les remous d'un passé qui refait surface dans des histoires souvent liées, par le dispositif de l'enquête policière (qui est aussi bien sûr aussi un dispositif littéraire), à la mise au grand jour des crimes perpétrés dans le passé par le régime d'apartheid.

Les genres peut être moins vendeurs en France de l'anticipation et de la science-fiction à la sud-africaine connaissent aussi depuis la fin des années 2000 un succès international, et questionnent d'une autre manière le lien entre passé et présent de l'Afrique du Sud. Le plus connu de ces romans est certainement le Zoo city de Lauren Beukes, auteur originaire du Cap, qui a connu un succès immédiat à la sortie de ce second roman en 2010. Preuve de son succès mondial, l'auteure s'est vue décerner le prestigieux prix Arthur Clarke ${ }^{4}$ en 2011, ce qui en a précipité la traduction en plusieurs langues, dont le français, et sa publication chez Eclipse en 2011. Lauren Beukes est en fait assez représentative d'une nouvelle génération d'auteurs sud-africains. Son travail s'inscrit dans une série de petits collectifs artistiques formés entre Cape Town et Johannesburg et travaillant entre littérature, graphisme, design, animation, et fanzines $^{5}$ - voir à ce propos la couverture de Zoo City dessinée par le graphiste Joey Hifi, très actif dans le monde du design, des fanzines et de la bande-dessinée.

Couverture originale de Joe Hifi pour Zoo City, ici pour l'édition de poche française

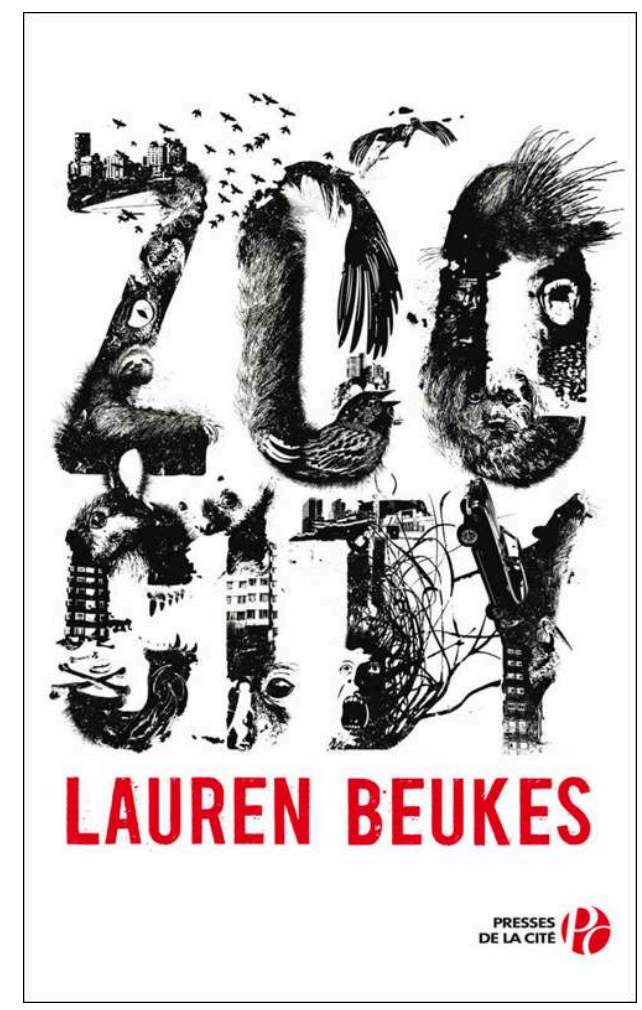


9 Ces collectifs inter-artistiques sont de petits mondes, où la pollinisation croisée est fréquente. Ils sont à l'origine de la montée en puissance locale du genre de la "Fiction Spéculative" en Afrique du Sud depuis la fin des années 2000 : ni tout à fait sciencefiction ni anticipation, ni non plus horreur, thriller, polar ou magie, la fiction spéculative met en scène des vampires, des fantômes, et autres sangomas ${ }^{6}$, au service de la création d'univers parallèles plus ou moins futuristes qui fonctionnent comme des méta-commentaires puissants décortiquant les dynamiques sociales de l'Afrique du Sud contemporaine. Fondamentalement urbain, ce genre emprunte à différents répertoires littéraires classiques eux aussi très citadins, en reprenant les codes du roman Noir de la grande tradition angelina qui mettait en scène dans les années 1940-1950 des détectives privés plus ou moins manipulés par leurs clients et plongés dans de longues enquêtes nébuleuses, prétextes à une dénonciation des turpitudes de Los Angeles (voir à ce sujet les analyses de Mike Davis, 1994).

10 Zoo City est un monument à plusieurs titres, d'abord par son utilisation de l'espace urbain de Johannesburg - et singulièrement du centre de la ville et de ses suburbs proches - qui, loin de n'être qu'un décor très finement décrit sur lequel se plaquerait l'action, participe d'une grande métaphore sur les recompositions et les inerties des frontières socio-spatiales sud-africaines à toutes les échelles. La capacité du roman à mélanger les genres, reprenant les grands éléments du réalisme magique latinoaméricain et les recettes du roman Noir américain, est tout aussi remarquable: le roman croise d'ailleurs plusieurs niveaux d'écriture, rapports de police, extraits de blogs, reportages qui entrecoupent l'action principale centrée sur une héroïne improbable, Zinzi December. Zinzi vit dans le quartier de Hillbrow à Johannesburg, dans un appartement qu'elle partage avec son compagnon, un Congolais qui, dit la légende, aurait fait le trajet à pied depuis Kinshasa, mais aussi un paresseux et une mangouste dont la présence nous est rapidement expliquée. Zinzi gagne sa vie en écrivant avec talent des scripts d'arnaques sur internet, et grâce à son don de découverte des objets perdus et personnes disparues. On pourrait donc dire qu'elle est un privé à la manière du Philip Marlowe de Raymond Chandler, et comme dans tout roman Noir qui se respecte, l'intrigue se centre rapidement sur une quête quand un producteur à succès la charge de retrouver l'un des deux jumeaux stars de la pop sudafricaine qui a disparu de sa résidence des riches quartiers Nord de Johannesburg dans des circonstances mystérieuses. Sur cette intrigue classique du roman Noir se greffe une intéressante dimension magique : Zinzi n'est pas exactement une enfant de chœur, et on découvre peu à peu son histoire et le secret de ses dons de voyance qui lui viennent de sa symbiose avec le paresseux quasi perpétuellement agrippé à ses épaules. Les circonstances de cette "animalisation" nous sont rapidement révélées, et Zinzi, on le comprend, traîne un lourd passé derrière elle : dans le monde de Zoo City, commettre un crime entraîne l'apparition d'un animal attitré pour le coupable, un animal-stigmate qui suit le coupable partout et qui lui confère aussi certains dons. N'importe quel animal, du moustique à l'ours polaire, plus difficile à cacher à qui n'est pas animalisé et Beukes s'offre une scène assez incroyable de cavale d'un gangster et de son ours dans les rues de Hillbrow. La réalité de la ville rejoint ici la fiction de cette version africaine du réalisme magique latino-américain: Hillbrow, le quartier de Johannesburg où se sont réfugiés tous les animalisés discriminés dans la société de Zoo City, endosse depuis la fin des années 1980 le rôle de quartier perdu de la vraie Johannesburg, tout à la fois symbole du déclin urbain, de la violence et du crime dans la grande histoire de la ville. Le quartier accueille par ailleurs une importante communauté étrangère, composée 
notamment de Nigérians et de Zimbabwéens. Le parallèle entre étrangers et animalisés court dans tout le roman, et pose la question plus générale du traitement des étrangers dans l'Afrique du Sud post-apartheid, dans le fort contexte xénophobe de ce pays qui lui vaut régulièrement la une de la presse internationale. Zoo City a d'ailleurs été écrit deux ans après l'une des plus fortes vagues d'attaques xénophobes qui ont eu lieu à travers tout le pays en 2008. Zinzi, contrainte de fuir à l'issue de son enquête et de ses révélations sur son puissant employeur, échappera à Zoo City à la toute fin du roman, en refaisant à l'envers le parcours des migrants africains attirés par la grande métropole d'Afrique australe, mais cette fois-ci ce cheminement est bien un cheminement vers la liberté :

"It's 4:30 am and the queue to the Beit Bridge ${ }^{7}$ border is already more than a thousand cars long, and that's on the South African side. Never mind the torrent of refugees trying to cross over from Zimbabwe. Barbed-wire fences barricade the dusty scrub on the riverbank from anyone stupid or desperate enough to try to swim across from Zimbabwe. After all, there are crocodiles in that river. The high drone of cicadas rises with the heat as we inch forward one car at a time through the carbon-monoxide fug. There is a bus two cars ahead of me loaded down on its axles with bags and chickens and a cram of people. The tangle of lost things on that bus swarms like a cloud of spagetti. And even here, there is that Zoo City hustle going on. Maybe it's not peculiar to Hillbrow. Maybe it's South Africa. You do what it takes, you take the opportunities" (Beukes, 2010, p. 307).

En ville, et malgré les frontières qui fragmentent Zoo City/Johannesburg, Zinzi est bien un personnage de la tradition chandlérienne: loin d'être cantonnée au quartier de Hillbrow comme beaucoup d'animalisés stigmatisés, elle arrive à circuler à travers tout Johannesburg au service de son enquête. Celle-ci l'amène, comme tout bon roman Noir qui se respecte, à dévoiler ce qui est caché, souterrain, et bien évidemment terrifiant, de la découverte d'indices dans les égouts de Johannesburg, à la scène finale épique l'opposant dans une cave au tueur en série animalisé d'un crocodile albinos qu'il nourrit de ses victimes, métaphore assez évidente de la période d'apartheid tout entière (voir sur le corps, le stigmate et le gothique dans les travaux de Lauren Beukes, les travaux de Mélanie Joseph-Vilain, 2014).

\section{La critique de la suburb comme critique de la société de consommation sud-africaine}

Deux autres romans méritent aussi le détour pour la force de la critique sociale et spatiale à laquelle ils renvoient. The Mall, de S.L. Grey est en fait écrit par deux auteurs de littérature de genre, Sarah Lotz et Louis Greenberg, qui ont combiné leurs deux noms. The Mall joue sur la limite toujours ténue dans l'Afrique du Sud suburbaine contemporaine entre le réel et le simulacre à la Baudrillard. En cherchant son inspiration dans le centre commercial, nouveau théâtre des rapports sociaux sudafricains, The Mall commente l'évolution consumériste de la société sud-africaine et les formes d'hypocrisie des rapports sociaux qu'elle produit. Le roman associe deux ratés de la Johannesburg contemporaine. Le premier, Dan, un jeune blanc de la classe moyenne johannesbourgeoise, peine à trouver sa voie dans la nouvelle Afrique du Sud. Employé du mall, il est en révolte contre sa condition d'Homo Suburbiensis :

"These fucking Johannesburg suburbs: they suck you in. They want you to get comfortable and complacent and enslaved just like the rest of the rats in this city. 
Working to pay for shit we don't need so that we can feel happy we've got a job"

(Grey, 2011, loc. 3237)

13 La deuxième protagoniste, Rhoda, est une jeune femme noire marginale expatriée en Grande-Bretagne et qui commence assez mal le roman en perdant dans le mall l'enfant dont elle avait la garde sous prétexte d'un rendez-vous avec son dealer. Elle et Dan se retrouvent rapidement prisonniers du centre commercial qui fonctionne comme un univers parallèle fortement hiérarchisé qui confronte les employés-esclaves (littéralement enchaînés à leur poste de travail) et les diverses catégories hiérarchisées de clients obsédés par la consommation, un mall dont il est impossible de s'échapper et qui transforme progressivement nos deux héros en monstres. Le roman est construit comme un séquençage de jeu vidéo, enchaînant des scènes d'interaction avec les employés et clients et des cheminements pénibles entre ces scènes, et en articulant spatialement les niveaux supérieurs "vitrines" du centre commercial et les sous-sols et corridors de services hantés par de gros monstres infects et par des individus déclassés qui ont perdu leur statut de consommateur privilégié du mall... Le sous-titre du roman, (downside), vient aussi souligner le renversement global dont procède l'ouvrage : on pourrait le traduire par "inconvénient", mais le mot évoque aussi le chamboulement absurde du cul-par-dessus-tête opéré par le roman qui place le shopping non plus comme moyen mais comme fin de l'existence. De fait, The Mall est assez drôle aussi, bourré de jeux de mots sur les noms des enseignes de toutes ces grandes chaînes spécialisées que l'on retrouve dans n'importe quel centre commercial du pays. Nos deux héros finissent tout de même par y échapper pour arriver à se réfugier dans la maison de la mère de Dan, mais pour mieux retourner dans le mall à la toute fin du roman, cette fois-ci poussés par l'anomie de la suburb johannesbourgeoise dont l'insipidité leur fait regretter leur vie parallèle finalement plus excitante. L'inversion entre les deux mondes, ou plutôt l'effacement des limites entre les deux, arrive finalement à l'issue du roman comme une évidence :

"We created a fantasy world for ourselves inside there, but this is the real world, outside, down below. The traffic jams, the flashing, seductive neon. That other place never existed; we can never escape. This is where we are" (Grey, 2011, loc. 4153).

14 La dernière ligne de dialogue du roman prononcée par Rhoda, "Game on", renvoie aussi le lecteur au défi du joueur entamant une nouvelle partie : cette fois-ci, ça se terminera peut-être mieux que la dernière fois?

15 Si la critique de la suburb, certes de moins en moins blanche mais toujours de la classe moyenne, est au cœur de plusieurs de ces romans de la fiction spéculative sud-africaine pour le consumérisme et l'aveuglement social dont font preuve ses résidents, Nineveh, le roman de Henrietta Rose-Innes publié en 2011, s'attaque aussi à la faillite écologique que représente ce modèle d'urbanisation. Dans Nineveh, Katya, exterminatrice et ellemême fille d'exterminateur, est chargée de désinsectiser un complexe sécurisé cossu de la Péninsule Sud du Cap qui semble infesté de goggas (cafards et autres petites bêtes en afrikaans). Comme les petites bêtes difficiles à voir en plein jour, le complexe est difficile à trouver, et Katya semble en permanence désorientée :

"Nineveh is so very new that it doesn't yet exist - not in the Cape Town street directory, and not on the maps in Katya's head. She stares at Zintle's map, but it's like a jigsaw piece for a picture she's never seen. She can't work out how these loops and forks correspond to any place real. When she tries to follow the route in her mind, she drifts into limbo: somewhere out past Noordhoek, between the new houses and the beach. Wetlands. Or so she thought" (Rose-Innes, 2011, loc.729). 
Nineveh répond donc bien à la volonté d'invisibilisation recherchée par ses résidents.

Mais la quête de Katya va au-delà : pour elle, retrouver les goggas, et surtout comprendre comment ils arrivent à entrer dans Nineveh, c'est aussi retrouver son propre passé, et l'histoire de ses parents... Le thème de la quête du détective est bien repris ici, mais ce n'est peut-être pas l'élément le plus marquant du roman. On est frappé finalement par la porosité de Nineveh : les goggas sont introuvables, mais ils doivent forcément passer quelque part? Surtout, entrent et sortent de Nineveh des objets, des bouts de matériaux de construction, des carreaux de céramique, revendus sur le bord de la route par les vendeurs informels des bidonvilles tout proches, eux aussi ordinairement invisibles... Il s'agit peut-être ici d'une référence aux histoires qui courent sur les domestiques sud-africains qui, selon la rumeur, voleraient chaque jour de petites quantités de sucre et de farine des réserves de leur patronne? Ou d'un commentaire plus ironique de l'auteur sur l'illusion de l'isolement, sur l'impossibilité de "sécuriser" complètement le complexe? Mais Nineveh procède aussi d'un commentaire empruntant au registre écologique : Nineveh envahie par les goggas est l'objet comme son homologue assyriennee de Ninive ${ }^{8}, d^{\prime}$ 'une "vengeance", cette fois-ci de la Nature, sur l'urbanisation des zones humides sensibles de la Péninsule du Cap. Mais on pourrait encore lire Nineveh autrement, et c'est le grand génie de l'ouvrage, en ce que le roman établit un parallèle entre la porosité des frontières posées dans l'espace de la Péninsule par Nineveh et la porosité plus générale des identités sud-africaines... qui renvoie elle-même à la porosité de la ville sud-africaine alors même que les formes de contrôle de la ville coloniale et d'apartheid visaient à en éliminer les situations de flou. L'impression donnée par un livre de photographies anciennes de la ville du Cap que feuillette Katya dans un mall à côté de Nineveh donne un bon exemple de ce malaise lié à la fluidité des situations urbaines :

"In none of the pictures does the city seem to be sitting easily with itself. Only the mountain and sea seem serene, altering at a far more dignified pace. A disorienting experience, looking at this book. Each person snapping the shutter had been trying to fix the city as it was, but there is no fixing such a shifting, restless thing as a discontented city. If you strung these pictures together in a giant flip-book, or put them together to make a jerky film reel, year on year, the city would be hopping and joggling, twitching and convulsing in a frenzy of urban ants-in-the-pants. Colonial cities are itchier than most, no doubt, fidgeting in the sub-Saharan light; harsh, even in a sepia world." (Rose-Innes, 2011, loc. 1451).

\section{Une rupture générationnelle par rapport à la littérature engagée anti-apartheid?}

Ces récentes aventures dans le champ de la fiction spéculative proposent de fait une rupture littéraire dans le paysage sud-africain, une rupture d'avec la littérature blanche engagée anti-apartheid traditionnelle représentée par Nadine Gordimer ou André Brinks, une rupture qui se joue donc bien sûr comme une rupture générationnelle. J'ai ainsi souvenir du Festival du Livre de Johannesburg en 2011 où Lauren Beukes, invitée d'honneur d'une table-ronde sur la fiction spéculative en compagnie de Sarah Lotz et quelques autres champions du genre, affirmait, non sans provoquer la polémique dans la salle, vouloir se libérer du poids de cette génération-là dans sa manière d'écrire et aussi dans les thématiques qu'elle souhaitait aborder dans ses romans. Le message semblait clair : ça suffit avec l'apartheid, il y a d'autres histoires 
à raconter sur l'Afrique du Sud. Mais cette émancipation n'est pas forcément si simple à atteindre: Angela Makholwa, jeune auteur à la fin des années 2000 d'un premier roman, le polar Red Ink, qui a connu un certain succès en Afrique du Sud, est très vite revenue sur le poids du passé et la difficulté à s'en libérer dans un entretien accordé en 2009: "The absence of apartheid rhetoric was deliberate because I thought South Africa had reached the political maturity to enjoy the kind of literature that is unburdened by our past. However, events of the past few months [NdA: elle fait référence ici aux attaques xénophobes de 2008] make me feel a bit like a school teacher who prescribes a 12th grade textbook to a group of third grade pupils. I'm no longer as certain of our ability to think outside of the racial boxes that we've been allocated. Perhaps we are not so ready after all, which would be profoundly sad"9.

On peut plutôt se demander s'il s'agirait ici, à travers la littérature de genre que représente la fiction spéculative, d'être moins sud-africain et plus universel dans le propos, et le succès mondial de Zoo City ainsi que les traductions progressives d'autres auteurs comme Henrietta Rose-Innes ou Sarah Lotz tendraient à prouver que cette stratégie de mise à distance du passé est efficace ${ }^{10}$. En tous les cas, si effet de génération il y a, tous ces romans procèdent d'emboîtements spatio-temporels qui, s'ils ne font pas directement référence au temps de l'apartheid grâce à l'abstraction historique que représente le genre de la fiction spéculative, renvoient de manière subtile aux inerties sociales et spatiales sud-africaines tout autant qu'aux transformations du moment contemporain par rapport aux temps de l'apartheid.

\section{BIBLIOGRAPHIE}

Brinks A., 1978. A dry white season. Londres, W.H. Allen. Traduit de l'anglais en français en 1980 par Robert Fouques-Duparc, Paris, Stock.

Davis M., 1994 [première édition 1990]. City of quartz: excavating the future in Los Angeles. Londres, Verso.

Despreez L.-F., 2008. Le noir qui marche à pied. Paris, Phébus.

Gordimer N., 1974. The conservationist. Londres, Jonathan Cape. Traduit de l'anglais en français en 1983 par Antoinette Roubichou-Stretz, Paris, Albin Michel.

Joseph-Vilain M., 2014. Cartographies génériques, spatiales et identitaires en Afrique du Sud : Margie Orford, Lauren Beukes, Henrietta Rose-Innes. Etudes Littéraires Africaines, 38, p. 69-82.

Makholwa A, 2007. Red Ink. Johannesburg, Picador Africa.

Meyer D., 2000. Orion. Le Cap, Human \& Rousseau. Traduit depuis la traduction anglaise en

français en 2003 par Robert Pépin sous le titre Les Soldats de l'aube, Paris, Le Seuil.

Middleton P., Woods T., 2000. Literatures of Memory: History, Time and Space in Post-war Writing. Mancheste, Manchester University Press.

Orford M., 2009. Daddy's Girl. Londres, Atlantic Books. Traduit de l'anglais en français en 2010 par Virginie Buhl, Paris, Payot. 
Sitographie (tous les sites ont été consultés en Décembre 2017) :

- Le lien vers le site de crowdfunding du projet Afro-SF: https://unglue.it/work/128685/\#

- Un extrait de lecture de Nineveh par Henrietta Rose-Innes : https://www.youtube.com/watch? $\mathrm{v}=\mathrm{RuYu}$ Dpd4KM

- Une interview en 2015 de Ivor Hartman : http://thisisafrica.me/lifestyle/storytimes-ivorhartmann-carrying-the-flag-for-independent-donor-free-african-publishers/ - L'interview de 2009 de Angela Makholwa : http://crimebeat.bookslive.co.za/blog/2008/03/12/ angela-makholwa-in-conversation-with-mike-nicol/

\section{NOTES}

1. La Science-Fiction ne représente bien évidemment qu'une des dimensions du renouvellement littéraire post-apartheid en Afrique du Sud. La place manque ici pour évoquer la multiplicité des genres, des auteurs et des sujets choisis.

2. Projet urbain associant centre d'affaires et résidences de luxe, édifié sur un polder et destiné aux classes supérieures et à la diaspora nigérianne. Pour un aperçu, voir le site https:// www.ekoatlantic.com/

3. En tous les cas, pour ceux qui écrivent dans ces langues et sont du coup traduits et publiés en France : l'un des auteurs sud-africains les plus lus dans son pays, Meshack Mfaniseni Masondo, a écrit et publié ses polars exclusivement en isizulu.

4. Le prix Arthur C. Clarke récompense chaque année depuis 1987 le meilleur roman de sciencefiction publié en Grande-Bretagne. Le prix a récompensé entre autres lauréats Margaret Atwood pour The Handmaid's tale en 1987, Amitav Ghosh en 1997 pour The Calcutta Chromosome et China Miéville pour The City and the City en 2010.

5. Voir notamment le fanzine sud-africain Something Wicked disponible en version Kindle.

6. Le sangoma est un genre de sorcier en Afrique du Sud.

7. Beit Bridge, ou Beitbridge (les deux graphies coexistent), est une ville-frontière entre l'Afrique du Sud et le Zimbabwe, et son pont un point de passage privilégié des voyageurs de la route reliant Johannesburg à Harare.

8. La chute de Ninive et du roi Sardanapale en 612 av. J.C. a été rapportée notamment par l'historien grec Ctésias de Cnide.

9. Interview sur http://crimebeat.bookslive.co.za/blog/2008/03/12/angela-makholwa-inconversation-with-mike-nicol / , site consulté en Novembre 2017.

10. Significativement d'ailleurs, Lauren Beukes a situé son roman suivant immédiatement Zoo City aux États-Unis, et l'a situé dans le genre classique du roman Noir américain.

\section{AUTEUR}

\section{SOPHIE DIDIER}

Sophie Didier, sophie.didier@u-pem.fr, est Professeur d'université, Université Paris Est Marne-laVallée, École d'Urbanisme de Paris et membre du Laboratoire Lab'Urba. 